\title{
Transitions induced by a magnetic field in slightly doped $\mathrm{TbMnO}_{3}$
}

Vera Cuartero, ${ }^{1,2}$ Javier Blasco, ${ }^{1 *}$ J. Alberto Rodríguez-Velamazán, ${ }^{1,3}$ Joaquín García, ${ }^{1}$ Gloria Subías $^{1}$ and Clemens Ritter. ${ }^{3}$

${ }^{1}$ Instituto de Ciencia de Materiales de Aragón, Departamento de Física de la Materia Condensada, CSIC-Universidad de Zaragoza. C/ Pedro Cerbuna 12, 50009 Zaragoza, Spain.

${ }^{2}$ ALBA Synchrotron Light Source, Cerdanyola del Vallès, Barcelona, 08290, Spain ${ }^{3}$ Institut Laue-Langevin, Boîte Postale 156, 38042 Grenoble Cèdex 9, France

Keywords: Multiferroic, Metamagnetism, Neutron diffraction, Competitive interactions, Ising ordering.

PACS numbers: 61.05.cp; 61.05.fm; 75.85.+t; 61.05.cj;64.70.Rh

\section{*Corresponding author:}

Javier Blasco

e-mail: jbc@posta.unizar.es; FAX: +34-976-761229.

Instituto de Ciencia de Materiales de Aragón

CSIC-Universidad de Zaragoza

50009 Zaragoza (Spain). 


\section{ABSTRACT}

We have investigated the magnetic properties of slightly doped multiferroic $\mathrm{TbMnO}_{3}$ after application of a magnetic field. The study focused on compositions $\operatorname{TbMn}_{1-\mathrm{x}} \mathrm{A}_{\mathrm{x}} \mathrm{O}_{3}$ $(\mathrm{x} \leq 0.1)$ with $\mathrm{A}=\mathrm{Ga}, \mathrm{Sc}, \mathrm{Co}$ and $\mathrm{Al}$. The replacement of $\mathrm{Mn}$ by $\mathrm{Ga}, \mathrm{Al}$ or $\mathrm{Sc}$ proved to be isovalent while the addition of Co leads to a partial charge transfer as $\mathrm{Mn}^{3+}+\mathrm{Co}^{3+} \rightarrow \mathrm{Mn}^{4+}+\mathrm{Co}^{2+}$. The samples with $10 \%$ of non-magnetic doping, $\mathrm{TbMn}_{0.9} \mathrm{Sc}_{0.1} \mathrm{O}_{3}, \quad \mathrm{TbMn}_{0.9} \mathrm{Al}_{0.1} \mathrm{O}_{3}$ and $\mathrm{TbMn}_{0.9} \mathrm{Ga}_{0.1} \mathrm{O}_{3}$, preserve the long range antiferromagnetic ordering of the Mn sublattice with, however, reduced transition temperatures compared to $\mathrm{TbMnO}_{3}$. New magnetic interactions in the Co-doped compound lead to the suppression of $\mathrm{Mn}$ ordering in $\mathrm{TbMn}_{0.9} \mathrm{Co}_{0.1} \mathrm{O}_{3}$. The application of an external magnetic field produces similar metamagnetic transitions in all $\mathrm{TbMn}_{0.9} \mathrm{~A}_{0.1} \mathrm{O}_{3}$ compounds that are ascribed to the Tb-sublattice. Powder neutron diffraction was used to determine the changes in the magnetic structure with applied magnetic field revealing a strong increase of $\mathrm{F}$ - and C-type magnetic reflections in these compounds. These results are accounted for by the anisotropic response of the $\mathrm{Tb}$ sublattice to a magnetic field while the Mn sublattice remains unchanged.

\section{Highlights:}

- We replace $\mathrm{Mn}$ by different cations in the multiferroic $\mathrm{TbMnO}_{3}$.

-We monitor the effects of substitution in macroscopic properties.

-We determine the magnetic ground state of the doped compounds.

- We identify metamagnetic transitions in the Tb sublattice.

- We model the magnetic ordering at high magnetic fields. 


\section{Introduction.}

$\mathrm{TbMnO}_{3}$ belongs to the family of magnetoelectric multiferroics [1] and undergoes on cooling three consecutive transitions [2]. The Mn sublattice orders antiferromagnetically (A-type) at $\mathrm{T}_{\mathrm{N} 1} \sim 41 \mathrm{~K}$. The order is incommensurate with a wave vector $\mathbf{k}_{\mathrm{Mn}}=\left(0, q_{\mathrm{Mn}} \sim 0.295,0\right)$ at $\mathrm{T}_{\mathrm{N} 1}$ [3]. The Mn moments are sinusoidally modulated and oriented along the $y$-direction $[3,4]$. On cooling, a spin rearrangement of the $\mathrm{Mn}$ moments yields an elliptical spiral in the $b c$-plane at $\mathrm{T}_{\mathrm{N} 2} \sim 27 \mathrm{~K}$. This transition is coupled to a ferroelectric phase transition with spontaneous polarization parallel to the $c$-axis [2]. Finally, the Tb-sublattice develops an incommensurate magnetic ordering at $\mathrm{T}_{\mathrm{N} 3} \sim 8 \mathrm{~K}$ with a different propagation vector $\mathbf{k}_{\mathrm{Tb}}=\left(0, q_{\mathrm{Tb}} \sim 0.42,0\right)[5]$.

Replacing Mn by a non-magnetic ion weakens the magnetic ordering of the Mnsublattice and is also detrimental to the ordering of the $\mathrm{Tb}$ moments and to the ferroelectric transition [6,7]. This fact reveals the great importance of the magnetic coupling between $\mathrm{Mn}$ and $\mathrm{Tb}$ moments, $\mathrm{J}_{\mathrm{Mn}-\mathrm{Tb}}$, for the occurrence of ferroelectricity in this system. Moreover, small substitutions affect only slightly the magnetoelectric properties of the Mn sublattice but influence strongly the magnetic ordering of the $\mathrm{Tb}$ moments suggesting that this ordering is determined by the competition between $\mathrm{J}_{\mathrm{Mn}-\mathrm{Tb}}$ and the direct coupling between $\mathrm{Tb}$ moments, $\mathrm{J}_{\mathrm{Tb}-\mathrm{Tb}}[6]$. A different behavior is expected for the replacement of $\mathrm{Mn}$ by $\mathrm{Co}$. This type of substitution is reported to be not isovalent in related systems [8,9] and more recently in the $\mathrm{TbMn}_{1-\mathrm{x}} \mathrm{Co}_{\mathrm{x}} \mathrm{O}_{3}$ series [10]. The couple $\mathrm{Mn}^{4+}-\mathrm{Co}^{2+}$ is more stable than the couple $\mathrm{Mn}^{3+}-\mathrm{Co}^{3+}$ in the perovskite structure and hence, this dopant can be magnetic $\left(\mathrm{Co}^{3+}\right.$ may adopt the non-magnetic low spin configuration in a perovskite lattice). To a first approximation, the ionic formula for the transition metals in $\mathrm{TbMn}_{0.9} \mathrm{Co}_{0.1} \mathrm{O}_{3}$ would be $\mathrm{TbMn}_{0.8}{ }^{3+} \mathrm{Mn}_{0.1}{ }^{4+} \mathrm{Co}_{0.1}{ }^{2+} \mathrm{O}_{3}$ and the partial addition of Co may lead to competitive magnetic interactions such as the 
ferromagnetic (FM) superexchange $\mathrm{Mn}^{4+}-\mathrm{O}-\mathrm{Co}^{2+}$, the $\mathrm{FM}$ double exchange $\mathrm{Mn}^{4+}-\mathrm{O}-$ $\mathrm{Mn}^{3+}$ and the rest of antiferromagnetic (AFM) superexchange interactions: $\mathrm{Co}^{2+}-\mathrm{O}-$ $\mathrm{Co}^{2+}, \mathrm{Mn}^{3+}-\mathrm{O}-\mathrm{Mn}^{3+}$ and $\mathrm{Mn}^{4+}-\mathrm{O}-\mathrm{Mn}^{4+}[10,11]$.

The magnetic interactions of these materials can be influenced as well by a magnetic field. The existence of metamagnetic transitions in Tb-based perovskites $[12,13]$ including $\mathrm{TbMnO}_{3}$ are well known [14]. These transitions were ascribed to the strong anisotropy of $\mathrm{Tb}^{3+}$ ions whose spins behave as Ising-like ones confined into the $a b$-plane [15]. The replacement of $\mathrm{Mn}$ by other cations in $\mathrm{TbMnO}_{3}$ strongly affects the magnetic coupling between both sublattices $\left(\mathrm{J}_{\mathrm{Mn}-\mathrm{Tb}}\right)$ In this way, we expect the presence of similar field induced magnetic transitions in doped $\mathrm{TbMnO}_{3}$ but the influence of dopant is unknown. The aim of this work is thus the investigation of the magnetic field effects on the compounds $\mathrm{TbMn}_{1-\mathrm{x}} \mathrm{A}_{\mathrm{x}} \mathrm{O}_{3}(\mathrm{x} \leq 0.1)$ with non-magnetic $(\mathrm{A}=\mathrm{Al}, \mathrm{Sc}$ and $\mathrm{Ga})$ and magnetic $(\mathrm{A}=\mathrm{Co})$ dopants. Magnetization measurements and powder neutron diffraction have been used for this purpose. We found that despite having different magnetic ground states at zero fields these samples present similar metamagnetic transitions which are caused by the magnetic transitions of the Tb-sublattice. The magnetism present in the Mn-sublattice remains unaltered by the external magnetic field up to $5 \mathrm{~T}$. These results suggest that $\mathrm{J}_{\mathrm{Tb}-\mathrm{Tb}}$ is much more sensitive to the strength of the magnetic field than $\mathrm{J}_{\mathrm{Mn}-\mathrm{Mn}}$. Finally, we have determined the magnetic arrangement of $\mathrm{Tb}^{3+}$ moments from the symmetry analysis and the geometry of the diffraction measurement.

\section{Experimental section.}

All the samples have been synthesized by solid state chemistry reaction. Stoichiometric amounts of $\mathrm{Tb}_{4} \mathrm{O}_{7}, \mathrm{MnCO}_{3}$ and the corresponding $\mathrm{Ga}_{2} \mathrm{O}_{3}, \mathrm{Sc}_{2} \mathrm{O}_{3}$ or $\mathrm{Co}_{3} \mathrm{O}_{4}$ were mixed and heated at $1000^{\circ} \mathrm{C}$ for $12 \mathrm{~h}$ in air. The resulting powders were 
pressed into pellets and sintered at $1200^{\circ} \mathrm{C}$ for $24 \mathrm{~h}$ also in air. The last steps consisted on repressing and sintering the pellets at $1400^{\circ} \mathrm{C}$ for 2 days in argon flow. The chemical composition of the samples was tested using a wavelength dispersive x-ray fluorescence spectrometer (Advant'XP+ from Thermo-ARL) and no significant differences were found between experimental and calculated compositions. X-ray diffraction patterns have been collected at room temperature using a Rigaku $\mathrm{D} / \mathrm{max}-\mathrm{B}$ diffractometer with a copper rotating anode and a graphite monochromator in order to select the $\mathrm{Cu} \mathrm{K} \alpha$ wavelength $(\lambda=1.5418 \AA)$. The patterns were collected in a range $18^{\circ} \leq$ $2 \theta \leq 130^{\circ}$ with a step $\Delta \theta=0.03^{\circ}$. The x-ray powder diffraction patterns of all samples agree with a perovskite single phase adopting the expected orthorhombic structure with Pbnm space group.

Neutron diffraction measurements were carried out at the Institut Laue-Langevin (ILL) in Grenoble (France) using two powder diffractometers. A preliminary study was performed on all $\mathrm{TbMn}_{1-\mathrm{x}} \mathrm{A}_{\mathrm{x}} \mathrm{O}_{3}(\mathrm{x}=0.05,0.1 ; \mathrm{A}=\mathrm{Al}, \mathrm{Co}, \mathrm{Ga}$ and $\mathrm{Sc})$ compounds using the high flux powder diffractometer D1B between 80 and $2 \mathrm{~K}$ in an angular range $1^{\circ} \leq$ $2 \theta \leq 127^{\circ}$ with $\Delta \theta=0.1^{\circ}$ using a wavelength of $2.52 \AA$. A magnetic study was carried out for $\mathrm{TbMn}_{0.9} \mathrm{Co}_{0.1} \mathrm{O}_{3}$ on this instrument with patterns collected at selected temperatures between 2 and $130 \mathrm{~K}$, and at magnetic fields up to $30 \mathrm{KOe}$. Powder specimens of $\mathrm{TbMn}_{0.9} \mathrm{Sc}_{0.1} \mathrm{O}_{3}$ and $\mathrm{TbMn}_{0.9} \mathrm{Ga}_{0.1} \mathrm{O}_{3}$ were measured on the high intensity diffractometer D20 in an angular range $4^{\circ} \leq 2 \theta \leq 130^{\circ}$ with $\Delta \theta=0.1^{\circ}$ using a wavelength of $2.40 \AA$. The patterns were collected in a temperature range between 2 and $60 \mathrm{~K}$, and in a magnetic field range between 0 and $50 \mathrm{KOe}$. The refinement program used was FULLPROF [16], both for x-ray and neutron diffraction measurements.

The temperature dependence of the dc magnetization and the ac magnetic susceptibility were measured on a commercial Quantum Design SQUID magnetometer 
from $2 \mathrm{~K}$ up to $295 \mathrm{~K}$. Magnetic hysteresis loops were collected at selected temperatures between -50 and $50 \mathrm{KOe}$.

\section{Results.}

\subsection{Macroscopic properties.}

All samples are isostructural as can be deduced from the x-ray patterns. They adopt the orthorhombic structure typical of $\mathrm{TbMnO}_{3}$ (Pbnm space group). Figure 1 shows the pattern of a representative sample. The substitution of Mn by another cation leads to changes in the unit cell size in accordance with the tabulated size of the inserted cation [17]. The inset of Fig. 1 shows the effect of these substitutions on the cell volume. Ga substitution produces the smallest change with a volume decrease of $\Delta \mathrm{V}=$ - $8.3 \AA^{3} / \mathrm{x}$ in this substitution range $(\mathrm{x} \leq 0.1)$. This is due to the great similarity between the ionic radii of $\mathrm{Ga}^{3+}(0.62 \AA)$ and $\mathrm{Mn}^{3+}(0.645 \AA)$. The greatest differences are obtained with the replacement of $\mathrm{Mn}^{3+}$ by $\mathrm{Sc}^{3+}(0.745 \AA)$ and $\mathrm{Al}^{3+}(0.535 \AA)$ giving rise to the largest volume expansion $\left(\Delta \mathrm{V}=+16.5 \AA^{3} / \mathrm{x}\right)$ and volume shrinkage $(\Delta \mathrm{V}=-22.5$ $\left.\AA^{3} / x\right)$, respectively. In the case of Co substitution, we observe a volume contraction ( $\Delta \mathrm{V}$ $=-18.9 \AA^{3} / \mathrm{x}$ ) similar to the Al substitution. This might be related with the addition of low spin (LS) $\mathrm{Co}^{3+}$ with a similar size to $\mathrm{Al}^{3+}$. However recent spectroscopic studies revealed that Co substitution is not isovalent, with an incomplete charge transfer from $\mathrm{Mn}^{3+}$ to $\mathrm{Co}^{3+}$ yielding a mixed valence character to both cations and the formal equilibrium $\mathrm{Mn}^{3+}+\mathrm{Co}^{3+} \leftrightharpoons \mathrm{Mn}^{4+}+\mathrm{Co}^{2+}$ shifted to the right [10]. Therefore the contraction observed on Co substitution may also be ascribed to the formation of small $\mathrm{Mn}^{4+}$.

This type of substitution, although very small, strongly affects the properties of $\mathrm{TbMnO}_{3}$. Fig. 2 shows the heat capacity $\left(\mathrm{C}_{\mathrm{p}}\right)$ measurements of these samples. The temperature dependence of $\mathrm{C}_{\mathrm{p}} / \mathrm{T}$ of $\mathrm{TbMnO}_{3}$ shows three anomalies as previously 
reported [2]. On cooling, they correspond to the magnetic ordering of $\mathrm{Mn}^{3+}$ moments $\left(\mathrm{T}_{\mathrm{N} 1}=41.3 \mathrm{~K}\right)$, the magnetic rearrangement of $\mathrm{Mn}^{3+}$ coupled to the ferroelectric transition $\left(\mathrm{T}_{\mathrm{N} 2}=\mathrm{T}_{\mathrm{FE}}=26.1 \mathrm{~K}\right)$ and the magnetic ordering of $\mathrm{Tb}^{3+}$ moments $\left(\mathrm{T}_{\mathrm{N} 3}=6.5 \mathrm{~K}\right)$. All substitutions are detrimental to the three transitions but the exact change depends on the nature of the dopant cation. $\mathrm{T}_{\mathrm{N} 1}$ decreases with increasing the concentration of nonmagnetic cations. This is an expected result but for a given concentration, the $\mathrm{T}_{\mathrm{N} 1}$ diminution depends on the dopant size in the way $\mathrm{T}_{\mathrm{N} 1}{ }^{\mathrm{Al}}>\mathrm{T}_{\mathrm{N} 1}{ }^{\mathrm{Ga}}>\mathrm{T}_{\mathrm{N} 1} \mathrm{Sc}$. The small size of $\mathrm{Al}^{3+}$ seems to be less unfavorable to the ordering of $\mathrm{Mn}^{3+}$ moments than the small size mismatch of $\mathrm{Ga}^{3+}$ atoms. $\mathrm{Sc}^{3+}$, with the largest ionic radius and a large size mismatch, is the most detrimental to the Mn order among non-magnetic dopants. In the case of Co substitution, the antiferromagnetic ordering of $\mathrm{Mn}$ is strongly weakened for $\mathrm{x}=0.05$ and it vanishes for $\mathrm{x}=0.1$. This is likely due to the mixed valence character of $\mathrm{Mn}$ and Co cations yielding competitive magnetic interactions which in addition to the induced structural disorder are very detrimental to the long range AFM ordering of $\mathrm{Mn}^{3+}$ moments.

The effect of these substitutions on the magnetic ordering of $\mathrm{Tb}^{3+}$ is more surprising. The intensity of the peak ascribed to this transition (and the concomitant entropy content) strongly decreases for any of these substitutions. The entropy content follows the sequence $\mathrm{Al}>\mathrm{Ga}>\mathrm{Sc}>\mathrm{Co}$. Again, there is a relationship to the dopant size and the isovalent replacement. For $\mathrm{x}=0.1$ samples, the peaks become more rounded and the shape resembles a Schottky anomaly (Figure 2b). This result reveals the strong correlation between the magnetic ordering of both sublattices. Any perturbation in the magnetic arrangement of the Mn-sublattice strongly influences the ordering of the Tbsublattice. 
Similar results are observed for the ferroelectric transition at $\mathrm{T}_{\mathrm{N} 2}$. For $\mathrm{x}=0.05$ samples, the temperature of the anomaly decreases, with $\mathrm{T}_{\mathrm{N} 2}{ }^{\mathrm{Al}}>\mathrm{T}_{\mathrm{N} 2}{ }^{\mathrm{Ga}}$, and no clear anomalies are noticeable for Sc- and Co-doped samples. For $\mathrm{x}=0.1$, there is no peak specific for this transition in any of the samples. However, such peaks if present could be screened by the wide Schottky anomaly. In this way, anomalies in the dielectric constant have been reported for $\mathrm{TbMn}_{0.9} \mathrm{Ga}_{0.1} \mathrm{O}_{3}$ and $\mathrm{TbMn}_{0.9} \mathrm{Al}_{0.1} \mathrm{O}_{3}$. In the case of the Ga doped compound a ferroelectric state develops [6] below $15 \mathrm{~K}$, while in the second sample a behavior typical of a relaxor has been found below $20 \mathrm{~K}$ [18]. These results agree with the importance of the non-linear magnetic structure of Mn-moments to develop ferroelectricity in Tb-based manganites [19]. In sample where this ordering is strongly weakened or vanished, there is not transition related to the ferroelectric transition in $\mathrm{C}_{\mathrm{p}}$ curves.

The magnetic susceptibility of these samples has been already reported [10]. All compounds show a paramagnetic behavior at high temperature and the Curie-Weiss law is obeyed above $100 \mathrm{~K}$ with the expected contribution from the paramagnetic cations. At low temperature, the susceptibility curves show a peak at $\sim 5 \mathrm{~K}$ indicating the occurrence of magnetic ordering in the $\mathrm{Tb}$ sublattice. The only different behavior concerns $\mathrm{TbMn}_{0.9} \mathrm{Co}_{0.1} \mathrm{O}_{3}$ where spin-glass-like properties are observed [10].

\subsection{Magnetic phase at zero field.}

The magnetic ground state was studied by powder neutron diffraction. Figure 3 shows the neutron patterns collected at $2 \mathrm{~K}$ for these samples. The compounds doped with non-magnetic cations exhibit similar patterns. Superstructure peaks arising from the antiferromagnetic ordering of the Mn moments are noticeable for $\operatorname{TbMn}_{1-\mathrm{x}} \mathrm{A}_{\mathrm{x}} \mathrm{O}_{3}$ $(\mathrm{x}=0.05,0.1 ; \mathrm{A}=\mathrm{Al}, \mathrm{Ga}, \mathrm{Sc})$. The incommensurate propagation vector agrees with $\mathbf{k}_{\mathrm{Mn}}=$ $(0, \sim 1 / 4,0)$ and the intensity of the magnetic peaks is weaker for the sample doped with 
$\mathrm{Sc}^{3+}$. Regarding the substitution with $\mathrm{Co}$, the magnetic peaks are even weaker in $\mathrm{TbMn}_{0.95} \mathrm{Co}_{0.05} \mathrm{O}_{3}$ and no sign of a magnetic ordering of the $\mathrm{Mn}$ sublattice is visible in $\mathrm{TbMn}_{0.9} \mathrm{Co}_{0.1} \mathrm{O}_{3}$ in agreement with the previous results from the $\mathrm{C}_{\mathrm{p}}$ measurements.

As already deduced from the heat capacity data the ordering of the $\mathrm{Tb}^{3+}$ moments is as well affected by the doping. In samples doped with non-magnetic cations the magnetic peaks ascribed to this ordering get more and more broadened in the sequence $\mathrm{Al}-\mathrm{Ga}-\mathrm{Sc}$ indicating an ordering getting more and more short ranged. At the same time the propagation vector of this magnetic order keeps the value of the modulation vector reported for $\mathrm{TbMnO}_{3}, \mathbf{k}_{\mathrm{Tb}}=(0, \sim 0.42,0)$. The increase in the doping ratio is very detrimental for the Tb-ordering and the associated peaks are strongly flattened for $\mathrm{x}=0.1$ samples, especially for the Sc-based one. This effect is even more drastic in the Cobased samples where one cannot longer see broad peaks but only an anomalous background at low angles ascribed to diffuse scattering whose origin is not clear so far. These results confirm the intimate relationship between the magnetic orderings of both sublattices $\left(\mathrm{Mn}^{3+}\right.$ and $\left.\mathrm{Tb}^{3+}\right)$ in $\mathrm{TbMnO}_{3}$. Any inhomogeneity in the Mn-O-Mn- chains strongly affects the occurrence of long range AFM ordering in the Tb sublattice.

\subsection{Metamagnetic transitions.}

A common characteristic in the Tb-based perovskites, including $\mathrm{TbMnO}_{3}$, is the existence of metamagnetic transitions $[12,13]$, i.e. spin rearrangements induced by an external magnetic field [20]. We have recently characterized the nature of this type of transition in a single crystal of $\mathrm{TbMn}_{0.9} \mathrm{Al}_{0.1} \mathrm{O}_{3}$ [18]. This section will be focused on the study of the metamagnetic transitions in $\mathrm{TbMn}_{0.9} \mathrm{~A}_{0.1} \mathrm{O}_{3}$ samples where $\mathrm{A}=\mathrm{Ga}, \mathrm{Sc}$ and Co. Figure 4 shows the magnetic hysteresis loops measured at $5 \mathrm{~K}$ for these samples. The isothermal magnetization shows an anomalous step at $\sim 2 \mathrm{~T}$ for all samples revealing a field induced magnetic transition. The shape is the same for these 
measurements independent of the existence (Sc- and Ga-doped samples) or nonexistence (Co-based sample) of a magnetic ordering of the Mn-sublattice. This fact suggests that the Tb-sublattice plays an important role in determining the metamagnetic transitions. Finally, the magnetic moment at $5 \mathrm{~T}$ is similar for $\mathrm{Ga}-$ and $\mathrm{Sc}-\mathrm{based}$ samples and slightly higher for the Co-doped sample. This effect may be ascribed to the additional paramagnetic contribution from $\mathrm{Mn} / \mathrm{Co}$-sublattice in the latter sample (lack of AFM order in the Mn-sublattice).

Neutron patterns were collected at different magnetic fields to shed light on the changes induced by the field in the magnetic properties. Selected patterns are displayed in Fig. 5. Similar changes are observed for the three samples. Under the effect of the magnetic field the diffuse scattering associated to the short range magnetic ordering of $\mathrm{Tb}^{3+}$ moments strongly decreases and for magnetic field values above $\sim 1.5 \mathrm{~T}$ the growth of some existing and the appearance of new magnetic peaks is induced. These new or growing peaks can be indexed with a propagation vector $\mathbf{k}=(0,0,0)$ and the most significant increase corresponds to C- and F-type reflections as can be seen in Fig. 6 where the dependence on the magnetic field for some reflections is shown. It is well known that magnetic ordering of Mn-moments with $\mathbf{k}=(0,0,0)$ can be described, using symmetry analysis, by four modes denoted as A, C, F and G [21]. Similar modes can be used for the Tb-ordering but it is worth remembering that this atom is placed in different point symmetry and has different extinction conditions. For example, the C-type ordering of Tb-moments also shows magnetic contribution in the $\left(\begin{array}{lll}0 & 1 & 1\end{array}\right)$ reflection whereas a FM ordering also contributes to the $\left(\begin{array}{lll}1 & 1 & 1\end{array}\right)$ reflection. Such reflections only correspond to G- and A-type, respectively, in the ordering of Mn-sublattice.

The reflections ascribed to the Mn-ordering remain unchanged up to fields of $5 \mathrm{~T}$ in $\mathrm{TbMn}_{0.9} \mathrm{Ga}_{0.1} \mathrm{O}_{3}$ and $\mathrm{TbMn}_{0.9} \mathrm{Sc}_{0.1} \mathrm{O}_{3}$ samples (see Fig. 6a). The field dependence of 
magnetic reflections ascribed to the Tb-order shows a similar trend for both types of doping (magnetic or not) as can be seen by comparing Fig. 6a and $6 \mathrm{~b}$. The temperature dependence of some magnetic peaks at $5 \mathrm{~T}$ is also shown in the Fig. 7. The magnetic Bragg peak reflecting the long range order of the Mn-sublattice appears in $\mathrm{TbMn}_{0.9} \mathrm{Sc}_{0.1} \mathrm{O}_{3}$ at $\sim 35 \mathrm{~K}$ in agreement with the anomaly observed in Fig. 2 under zero field conditions. This result reveals that magnetic fields up to $5 \mathrm{~T}$ do not affect significantly the magnetism of the Mn-sublattice. Regarding the magnetic reflections arising from the $\mathrm{Tb}$-sublattice, there is an enhancement in their intensity below $40 \mathrm{~K}$ but C-type reflections start to be noticeable at $\sim 60 \mathrm{~K}$. In the case of $\mathrm{TbMn}_{0.9} \mathrm{Co}_{0.1} \mathrm{O}_{3}$ less experimental points are available but the results are similar suggesting an onset for the C-type reflections between 70 and $50 \mathrm{~K}$.

In order to ascertain the type of magnetic ordering induced by the magnetic field, we have refined the neutron patterns taking into account the previous results in related compounds [13]. Due to a strong Ising anisotropy, $\mathrm{Tb}^{3+}$ moments are usually ordered in non-collinear structures in the $a b$-plane and the interaction with an external magnetic field changes the magnetic alignment. Due to this anisotropy, the final configuration of each grain in a polycrystalline sample strongly depends on its geometric orientation with respect to the magnetic field. This makes a quantitative study on polycrystalline samples very difficult but important qualitative information can still be extracted. A study on a $\mathrm{TbMn}_{0.9} \mathrm{Al}_{0.1} \mathrm{O}_{3}$ single crystal [18] with $\mathbf{H} \| \mathbf{b}$ revealed a metamagnetic transition of the $\mathrm{Tb}^{3+}$ moments. At high fields, they adopt the $\mathrm{C}_{\mathrm{x}} \mathrm{F}_{\mathrm{y}}$ structure following the Bertaut's nomenclature [21]. This implies that moments are ferromagnetically aligned along the y-direction while a C-type AFM ordering is observed for the $\mathrm{x}$ direction. This type of ordering also accounts for the occurrence of F- and C-type reflections in the neutron pattern at high fields for the present $\mathrm{TbMn}_{0.9} \mathrm{~A}_{0.1} \mathrm{O}_{3}$ samples 
$(\mathrm{A}=\mathrm{Sc}, \mathrm{Ga}$ or $\mathrm{Co})$. The ferromagnetic component along the field direction suggests that in the case of $\mathbf{H} \| \mathbf{a}$, the most plausible magnetic structure would be $\mathrm{F}_{\mathrm{x}} \mathrm{C}_{\mathrm{y}}$. Our attempts to refine the neutron patterns at high fields with a combination of both structures for $\mathrm{Tb}$ moments (shown in figure 8) yielded satisfactory results with the only exception of the lack of intensity for (100) and (010) reflections. The absence of these reflections in the experimental pattern can be understood taking into account the geometry of our experimental setup outlined in figure 9. The magnetic field is vertical and perpendicular to the scattering vector. When the diffraction condition is fulfilled for the $\left(\begin{array}{lll}1 & 0 & 0\end{array}\right)$ reflection —or the $\left(\begin{array}{lll}0 & 1 & 0\end{array}\right)$ one - in a particular crystallite, its scattering vector is perpendicular (not parallel) to the magnetic field and therefore the FM ordering along the scattering direction in very disfavored so that this crystallite cannot adopt the $\mathrm{F}_{\mathrm{x}} \mathrm{C}_{\mathrm{y}}$ magnetic structure - or the $\mathrm{C}_{\mathrm{x}} \mathrm{F}_{\mathrm{y}}$ type magnetic structure for the $\left(\begin{array}{lll}0 & 1 & 0\end{array}\right)$ case - and there is no magnetic contribution to this reflection.

Therefore, we have modeled the powder neutron patterns excluding the $2 \theta$ range of (010) and (100) reflections and including the magnetic contributions of the AFM ordering of the $\mathrm{Mn}^{3+}$ moments and of the two magnetic structures of the $\mathrm{Tb}^{3+}$ moments (Fig. 8a and 8b). For the Co-doped compound, only the magnetic contribution from the Tb-sublattice was considered (Fig. 8c). The refined magnetic moment for Mn atoms is 2.89(6) and 3.92(6) for $\mathrm{TbMn}_{0.9} \mathrm{Sc}_{0.1} \mathrm{O}_{3}$ and $\mathrm{TbMn}_{0.9} \mathrm{Ga}_{0.1} \mathrm{O}_{3}$ samples, respectively. These results suggest a more complete Mn-ordering in the Ga-based sample in agreement with its larger $\lambda$-anomaly in the Cp-measurements (see Fig. 2).

Regarding the ordering of $\mathrm{Tb}$ moments, only qualitative information can be extracted. Our refinement suggests a higher contribution from the $\mathrm{F}_{\mathrm{x}} \mathrm{C}_{\mathrm{y}}$ phase with respect to the $\mathrm{C}_{\mathrm{x}} \mathrm{F}_{\mathrm{y}}$ one. This may indicate that the Ising direction is closer to the a-axis. Previous studies in related systems $[12,13]$ found the Ising direction at $34-40^{\circ}$ from the 
a-axis in agreement with our hypothesis. Nevertheless, further studies on single crystals with different geometries are desirable to determine the Tb-ordering with accuracy.

\section{Conclusions.}

The substitution of $\mathrm{Mn}$ in $\mathrm{TbMnO}_{3}$ by small amounts of different cations leads in the case of non-magnetic atoms ( $\mathrm{Al}, \mathrm{Ga}, \mathrm{Sc})$ to a weakening of the Mn-O-Mn interaction with a gradual decrease of $\mathrm{T}_{\mathrm{N} 1}$. The weakening increases with increasing the cationic size of the non-magnetic dopant, but long range AFM ordering is preserved in the Mn-sublattice. In the Co-based sample, the non-isovalent substitution produces competitive magnetic interactions preventing the Mn ordering. All of these substitutions are very detrimental to the magnetic ordering of $\mathrm{Tb}$ moments observed in the parent $\mathrm{TbMnO}_{3}$ compound. Upon $10 \%$ of doping diffuse scattering is present in the neutron patterns indicating short range magnetic ordering of the Tb-moments. This indicates that a perfectly ordered magnetic Mn-sublattice is required to develop the incommensurate AFM ordering of $\mathrm{Tb}^{3+}$ observed in $\mathrm{TbMnO}_{3}$ [5]. This agrees with previous suggestions that this ordering arises from the interplay between $J_{\mathrm{Mn}-\mathrm{Tb}}$ and $\mathrm{J}_{\mathrm{Tb}}$ $\mathrm{Tb}$ interactions [22].

The application of external magnetic fields favors the $\mathrm{J}_{\mathrm{Tb}-\mathrm{Tb}}$ interaction and disturbs the coupling between $\mathrm{Mn}$ and $\mathrm{Tb}$ sublattices. While the Tb-sublattice gets realigned in the $a b$-plane the Mn-sublattice remains unchanged, i.e. with an AFM order in Ga- and Sc-based samples or disordered in the Co-doped sample $\left(\mathrm{J}_{\mathrm{Mn}-\mathrm{Mn}}\right.$ is practically insensitive to the magnetic field). The $\mathrm{Tb}^{3+}$ moments are ordered in a $\mathrm{F}_{\mathrm{i}} \mathrm{C}_{\mathrm{j}}$ mode $(\mathrm{i} \neq \mathrm{j}=\mathrm{x}, \mathrm{y})$ depending on the magnetic field orientation as the crystal field anisotropy fixes the direction of the moments $[12,13]$.

\section{Acknowledgements.}


The authors thank ILL for granting beam time at D20 and also to the Spanish CRG

D1B. Financial support from Spanish Spanish MEC (projects MAT2012-38213-C02-01 and MAT2011-27233-C02-02) and DGA (CAMRADS) is acknowledged.

\section{References.}

[1] N. A. Hill, J. Phys. Chem. B 104 (2000) 6694.

[2] K. Kimura, T. Goto, H. Shintani, K. Ishizka, T. Arima and Y. Tokura, Nature (London) 426 (2003) 55.

[3] S. Quezel, F. Tcheou, J. Rossat-Mignod, G. Quezel, E. Roudaut, Physica B 86 (1977) 916.

[4] J. Blasco, C. Ritter, J. García, J. M. De Teresa, J. Pérez-Cacho and M. R. Ibarra, Phys. Rev. B 62 (2000). 5609.

[5] R. Kajimoto, H. Yoshizawa, H. Shintani, T. Kimura and Y. Tokura, Phys. Rev. B 70 (2004) 012401.

[6] O. Prokhnenko, N. Aliouane, R. Feyerherm, E. Dudzik, A. U. B. Wolter, A. Maljuk, K. Kiefer and D. N. Argyriou, Phys. Rev. B 81 (2010) 024419.

[7] V. Cuartero, J. Blasco, J. García, G. Subías and M. C. Sánchez, J. Phys.: Conf. Series 200 (2010) 012024.

[8] D.V. Karpinsky, I.O. Troyanchuk, A.P. Sazonov, O.A. Savelieva and A. Heinemann, Eur. Phys. J. B 60 (2007) 273.

[9] B. Raveau and M. Seikh, 'Cobalt oxides from crystal chemistry to physics' Ed. Wiley-VCH (2012).

[10] V. Cuartero, J. Blasco, J. García, S. Lafuerza, G. Subías, J. A. RodriguezVelamazán and C. Ritter, J. Phys.:Condensed Matter 24 (2012) 455601.

[11] J. B. Goodenough, Phys. Rev. 100 (1995) 564; J. Kanamori, J. Phys. Chem. Solids 10 (1959) 87.

[12] J. Mareschal, J. Sivaridière, G. F. De Vries and E. F. Bertaut, J. Appl. Phys. 39 (1968) 1364.

[13] L. Holmes, R. Sherwood and L. G. Van Uitert, J. Appl. Phys 39 (1968) 1373.

[14] T. Kimura, G. Lawes, T. Goto, Y. Tokura and A. P. Ramírez, Phys. Rev. B 71 (2005) 224425. 
[15] S. Hüffner, L. Holmes, F. Varsanyi and L. G. Vanuiter, Phys. Lett. A 25 (1967) 301.

[16] J. Rodríguez-Carvajal, Physica B 192, 55 (1993); http://www.ill.eu/ sites/fullprof/

[17] R. D. Shannon, Acta Cryst. A 32 (1976) 751.

[18] V. Cuartero, J. Blasco, J. A. Rodríguez-Velamazán, J. García, G. Subías, C. Ritter, J. Stankiewicz, and L. Canadillas-Delgado, Phys. Rev. B 86 (2012) 104413.

[19] C. N. R. Rao, A. Sundaresan and R. Saha, J. Phys. Chem. Lett. 3 (2012) 2237.

[20] E. Stryjewsky and N. Giordano, Adv. Phys. 26 (1977) 487.

[21] E. F. Bertaut, Acta Cryst. A24 (1968) 217.

[22] O. Prokhnenko, R. Feyerherm, M. Mostovoy, N. Aliouane, E. Dudzik, A. U. B. Wolter, A. Maljuk and D. N. Argyriou, Phys. Rev. Lett. 99 (2007) 177206. 


\section{FIGURE CAPTIONS}

Figure 1. X-ray powder diffraction pattern for $\mathrm{TbMn}_{0.9} \mathrm{Al}_{0.1} \mathrm{O}_{3}$ at room temperature (points). The solid curve is the best fit from the Rietveld refinement while the bottom curve shows the difference between calculated and observed intensities and the vertical bars indicate the allowed Bragg reflections. Inset: Unit cell volume vs. dopant concentration for $\mathrm{TbMn}_{1-\mathrm{x}} \mathrm{A}_{\mathrm{x}} \mathrm{O}_{3}(\mathrm{x} \leq 0.1)$ samples. The type of dopant (A) is given for each curve.

Figure 2. Temperature dependence of the specific heat divided by temperature for (a) $\mathrm{TbMn}_{0.95} \mathrm{~A}_{0.05} \mathrm{O}_{3}$ and (b) $\mathrm{TbMn}_{0.9} \mathrm{~A}_{0.1} \mathrm{O}_{3}$ samples ( $\mathrm{A}=\mathrm{Al}, \mathrm{Ga}, \mathrm{Sc}$ and $\left.\mathrm{Co}\right)$.

Figure 3. Neutron powder diffraction at $2 \mathrm{~K}$ and zero magnetic field for (a) $\mathrm{TbMn}_{0.95} \mathrm{~A}_{0.05} \mathrm{O}_{3}$ and (b) and $\mathrm{TbMn}_{0.9} \mathrm{~A}_{0.1} \mathrm{O}_{3}$ samples $(\mathrm{A}=\mathrm{Al}, \mathrm{Ga}, \mathrm{Sc}$ and $\mathrm{Co})$. The patterns have been shifted vertically for the sake of comparison.

Figure 4. Magnetic hysteresis loops at $5 \mathrm{k}$ for $\mathrm{TbMn}_{0.9} \mathrm{Sc}_{0.1} \mathrm{O}_{3}, \mathrm{TbMn}_{0.9} \mathrm{Ga}_{0.1} \mathrm{O}_{3}$ and $\mathrm{TbMn}_{0.9} \mathrm{Co}_{0.1} \mathrm{O}_{3}$.

Figure 5. Neutron powder diffraction at $2 \mathrm{~K}$ and at different magnetic fields for (a) $\mathrm{TbMn}_{0.9} \mathrm{Sc}_{0.1} \mathrm{O}_{3}$, (b) $\mathrm{TbMn}_{0.9} \mathrm{Ga}_{0.1} \mathrm{O}_{3}$ and (c) $\mathrm{TbMn}_{0.9} \mathrm{Co}_{0.1} \mathrm{O}_{3}$.

Figure 6. Peak intensity of selected Bragg reflections vs. magnetic field intensity for (a) $\mathrm{TbMn}_{0.9} \mathrm{Sc}_{0.1} \mathrm{O}_{3}$ and (b) $\mathrm{TbMn}_{0.9} \mathrm{Co}_{0.1} \mathrm{O}_{3}$.

Figure 7. Peak intensity of Bragg reflections vs. temperature for (a) $\mathrm{TbMn}_{0.9} \mathrm{Sc}_{0.1} \mathrm{O}_{3}$ and (b) $\mathrm{TbMn}_{0.9} \mathrm{Co}_{0.1} \mathrm{O}_{3}$.

Figure 8. Rietveld refinements of the neutron powder diffraction at $2 \mathrm{~K}$ and $\mu_{0} \mathrm{H}=3 \mathrm{~T}$ for (a) $\mathrm{TbMn}_{0.9} \mathrm{Sc}_{0.1} \mathrm{O}_{3}$, (b) $\mathrm{TbMn}_{0.9} \mathrm{Ga}_{0.1} \mathrm{O}_{3}$ and (c) $\mathrm{TbMn}_{0.9} \mathrm{Co}_{0.1} \mathrm{O}_{3}$.

Figure 9. Geometry in the diffraction measurement at D1B/D20. Magnetic field is located vertically, ie perpendicular to the plane composed by scattering vector $\mathbf{Q}$ and both the incoming and outgoing neutron beam (indicated by the vectors $\mathbf{k}$ ). Left and right pictures show the arrangement for $\left(\begin{array}{lll}0 & 1 & 0\end{array}\right)$ and $\left(\begin{array}{lll}1 & 0 & 0\end{array}\right)$ planes, respectively. 
Figure 1.

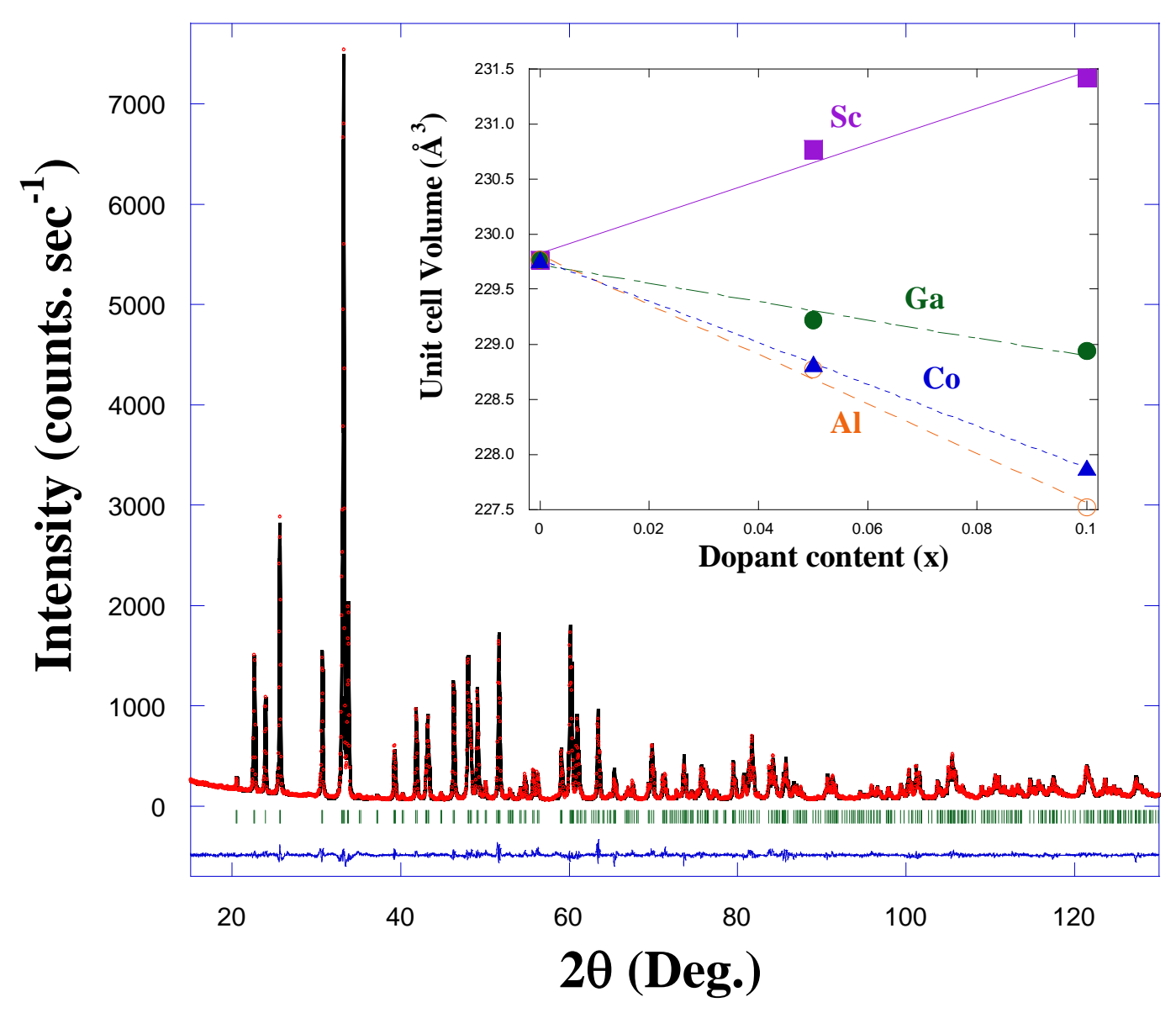


Figure 2.

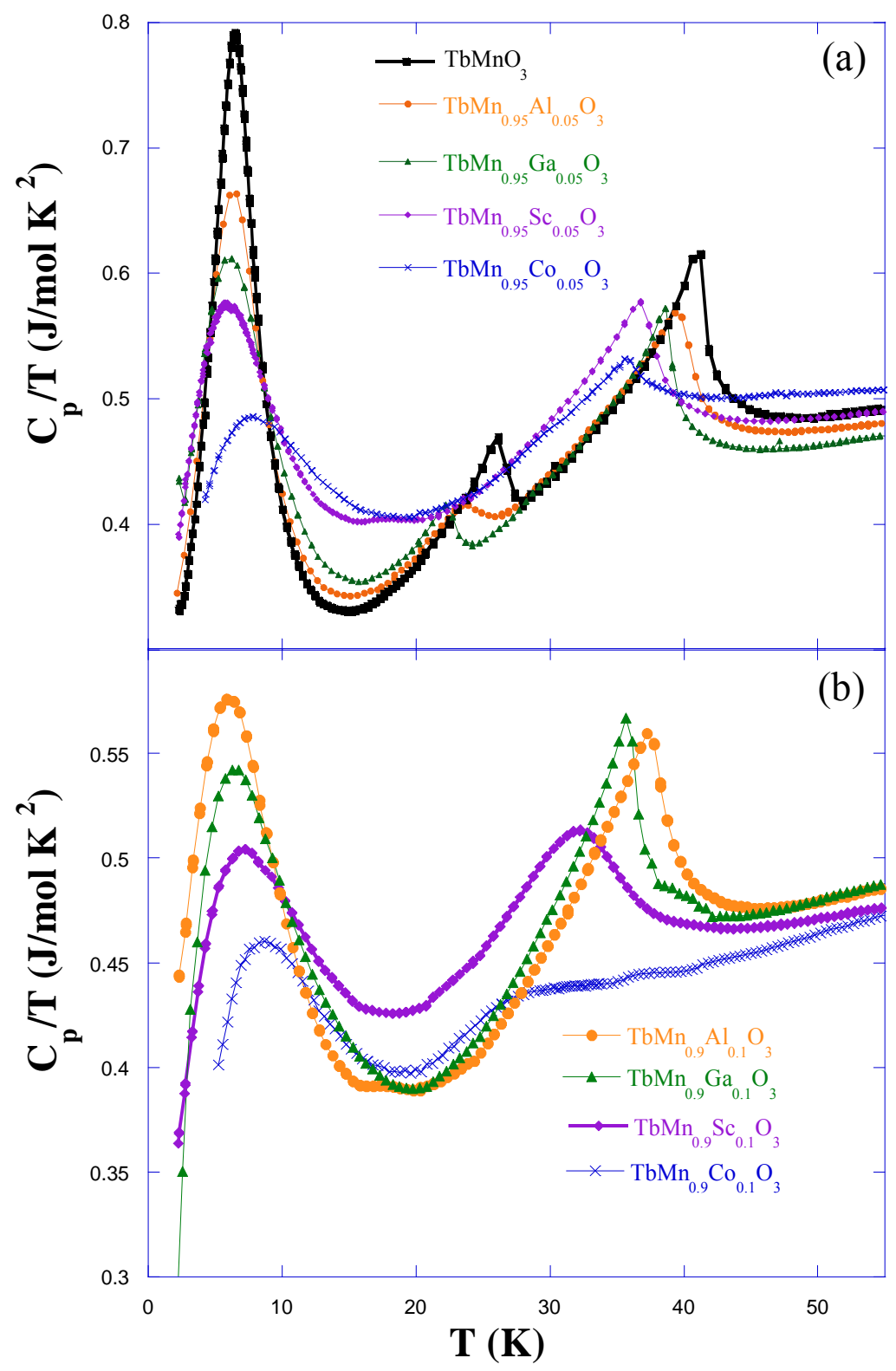


Figure3.

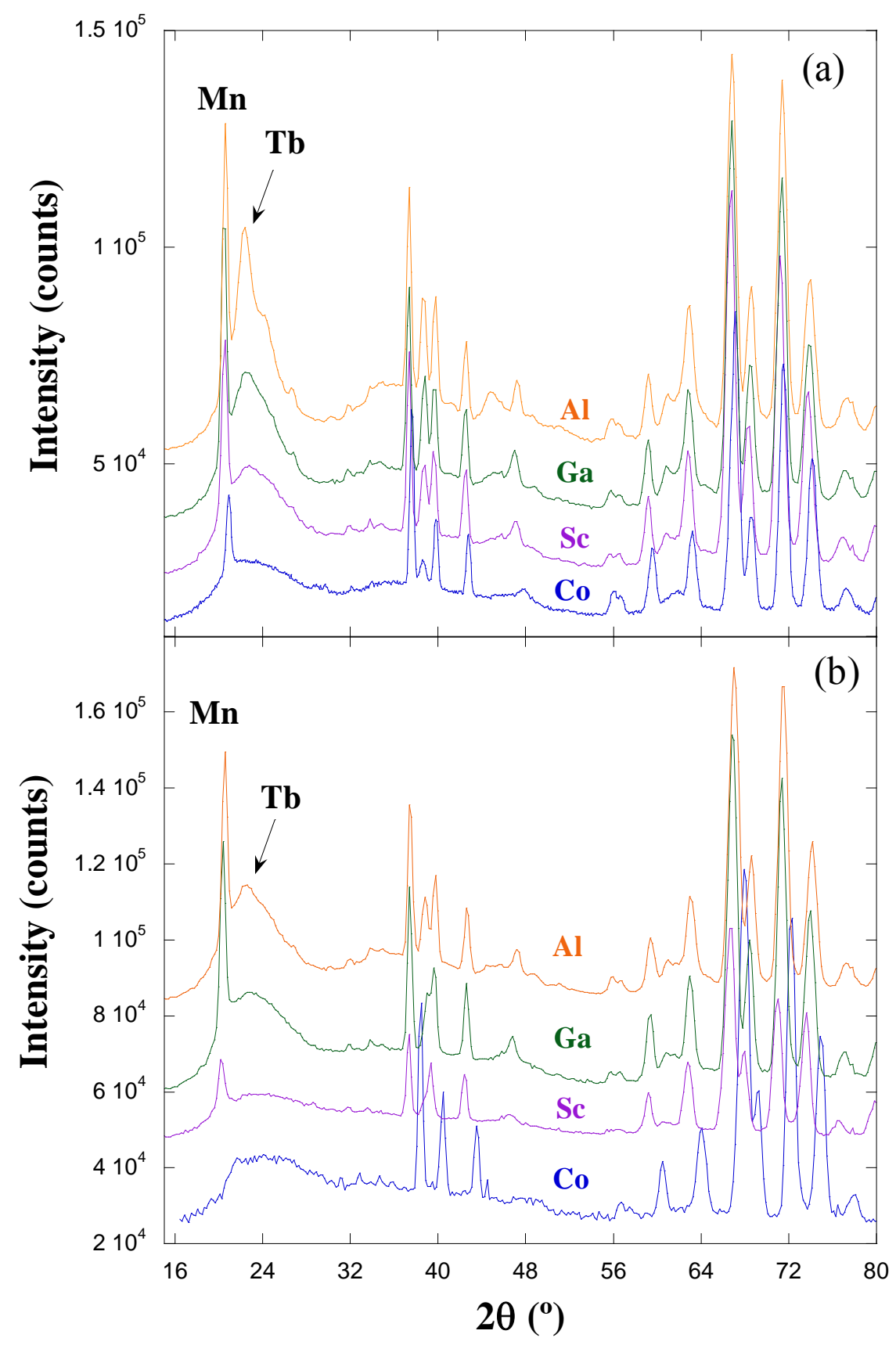


Figure 4.

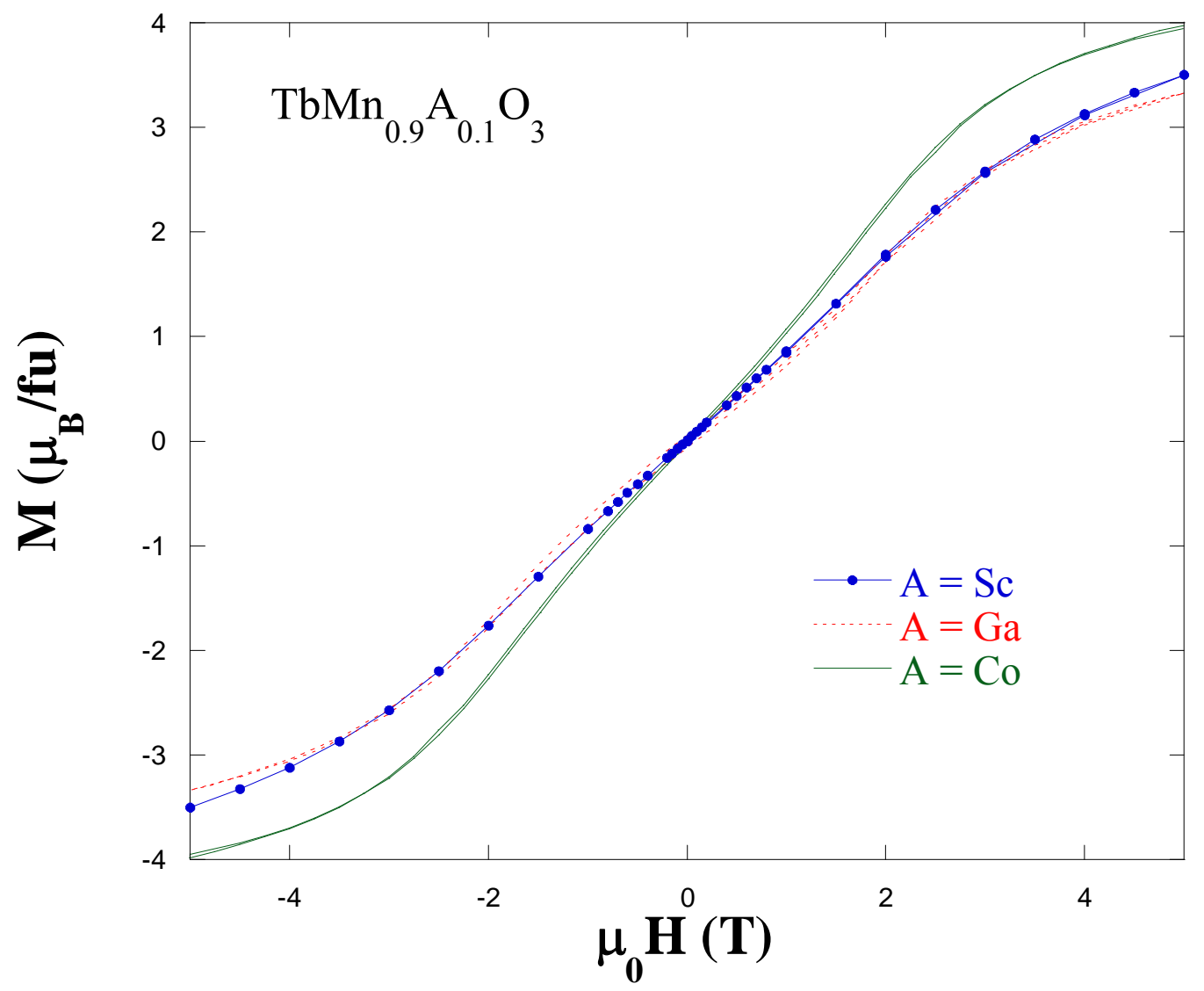


Figure 5.

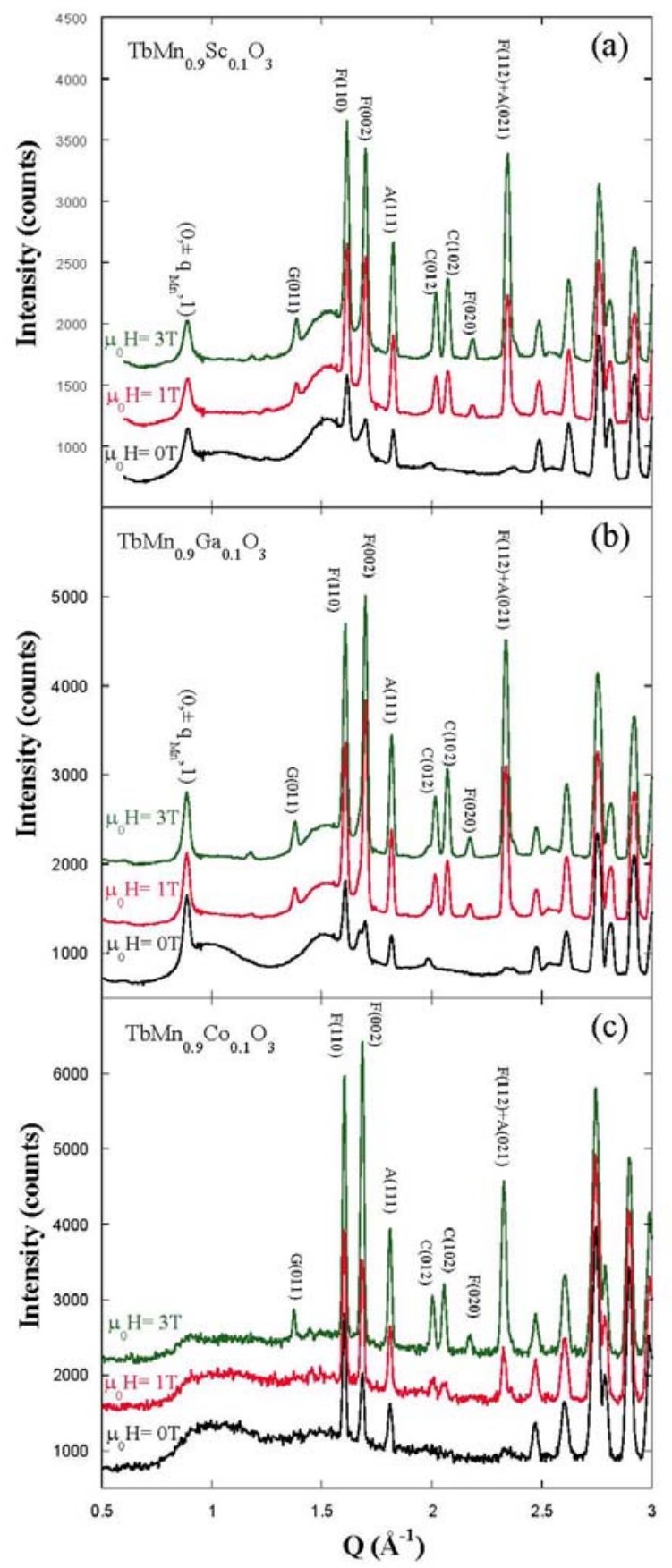


Figure 6.
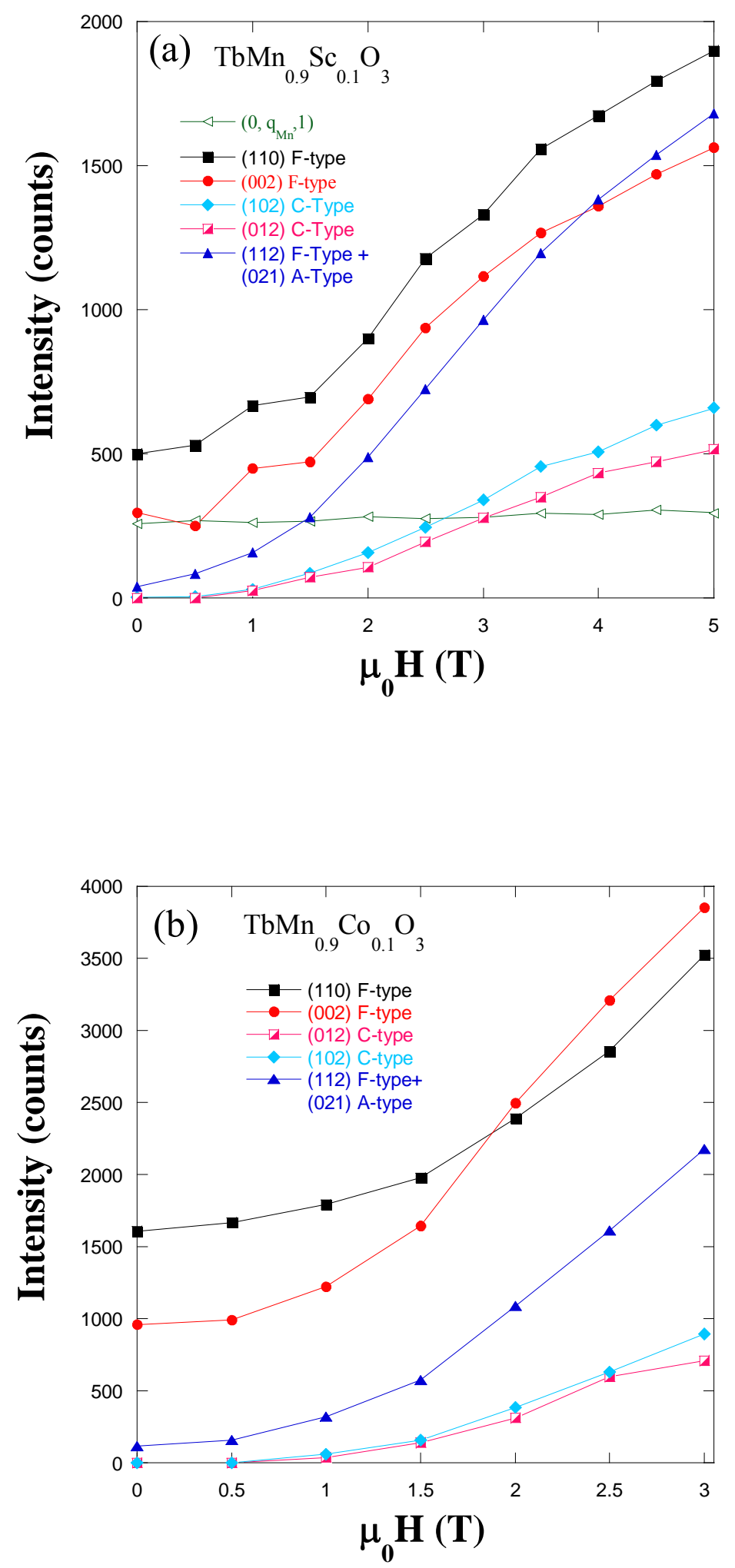
Figure 7.
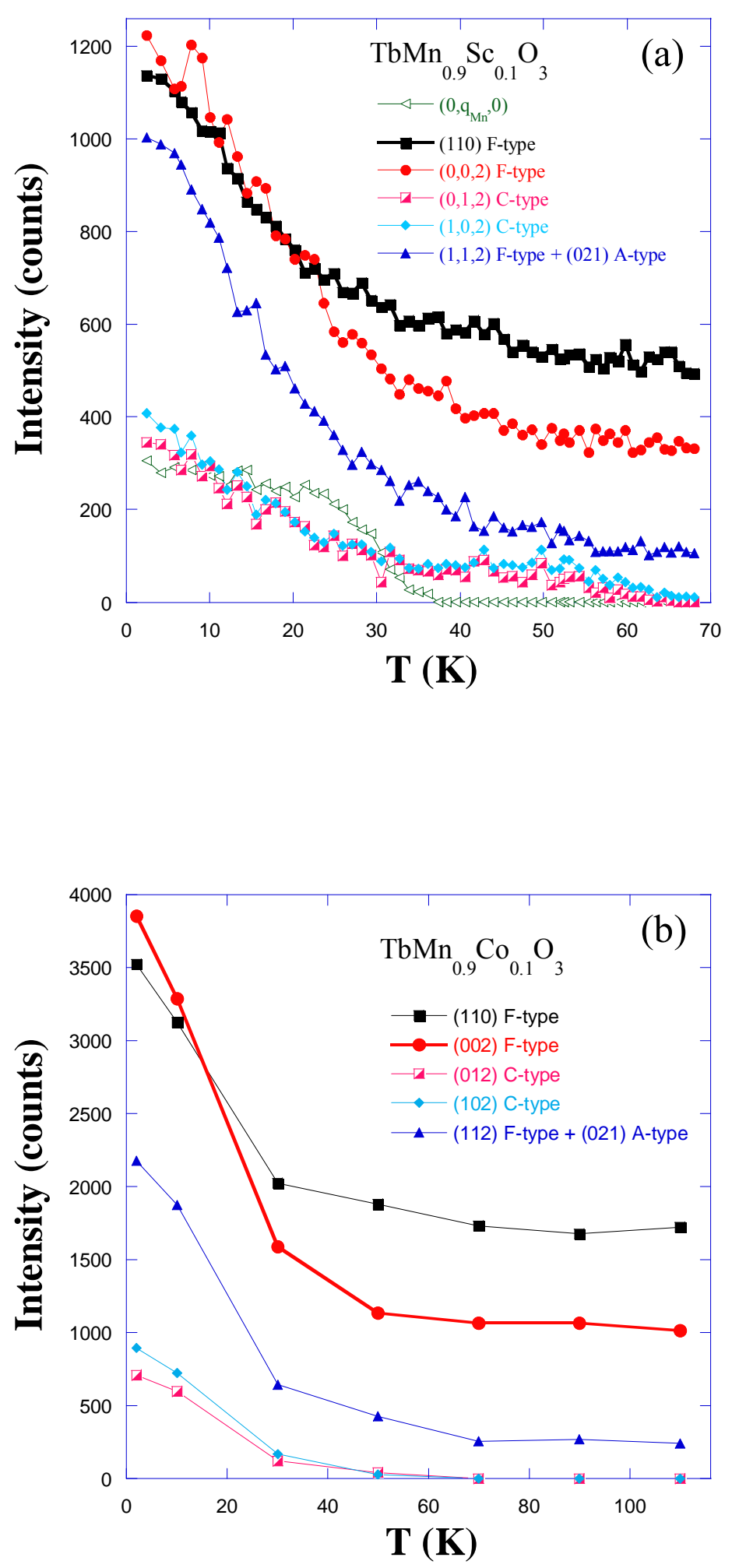
Figure 8.

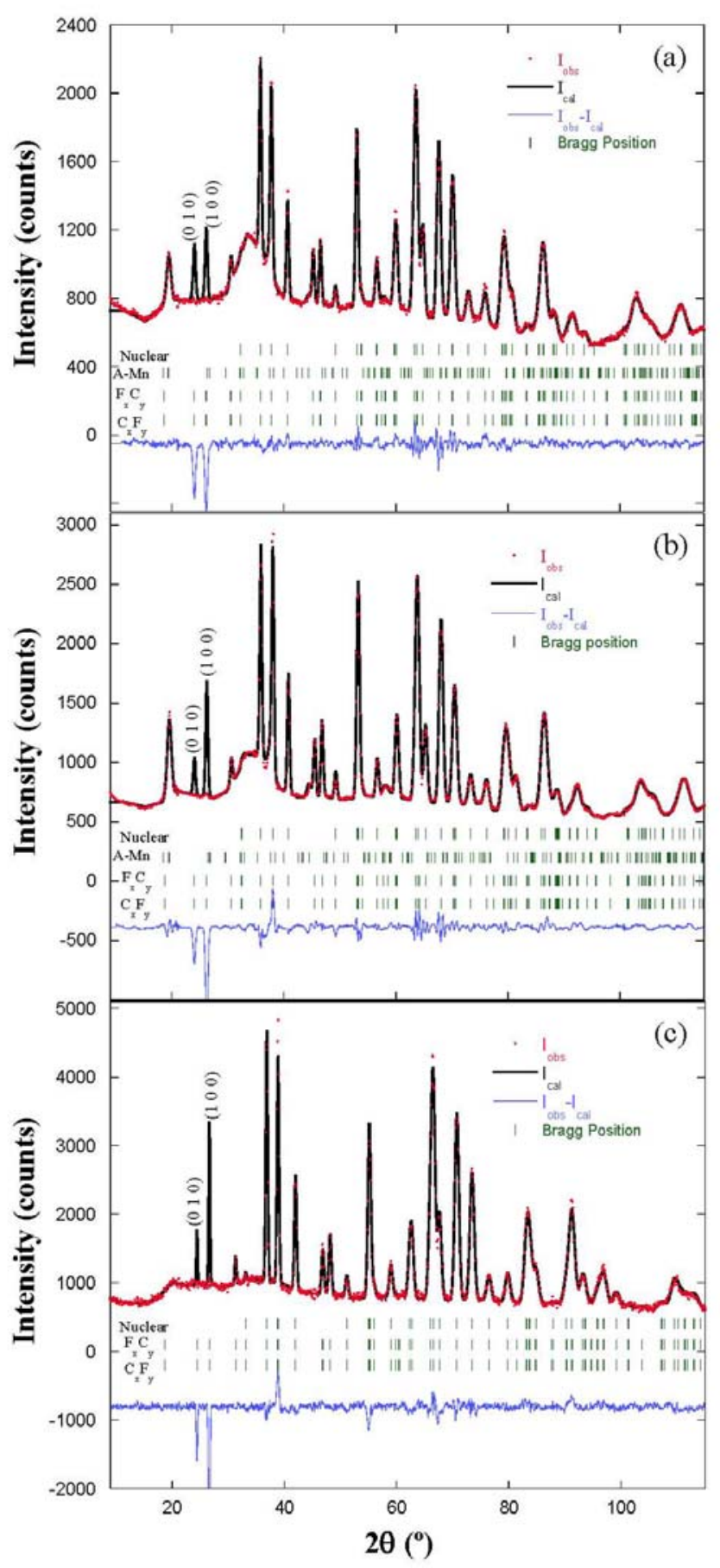


Figure 9.
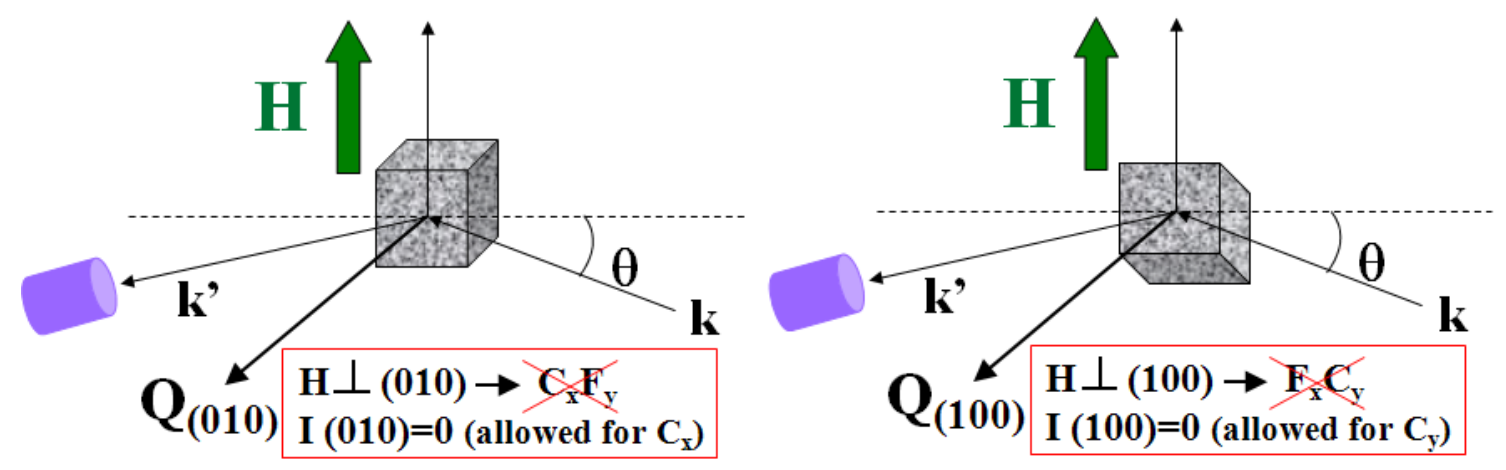